\title{
Retention in Care and Adherence to ART are Critical Elements of HIV Care Interventions
}

\author{
Sebastian M. Stricker $\cdot$ Kathleen A. Fox • \\ Rachel Baggaley $\cdot$ Eyerusalem Negussie $\cdot$ \\ Saskia de Pee $\cdot$ Nils Grede $\cdot$ Martin W. Bloem
}

Published online: 29 November 2013

(c) Springer Science+Business Media New York 2013

\begin{abstract}
Retention in care and adherence to antiretroviral treatment (ART) are critical elements of HIV care interventions and are closely associated with optimal individual and public health outcomes and cost effectiveness. This literature review was conducted to analyse how the roles of clients in HIV care and treatment are discussed, from terminology used to measurement methods to consequences of a wide range of patient-related factors impacting client adherence to ART and retention in care. Unfortunately, data suggests that clients find it hard to follow recommended behaviour. For HIV, the greatest loss to follow-up occurs before starting treatment, though each step of the continuum of care is affected. Measurement approaches can be divided into 'direct' and 'indirect' methods; in practice, a combination is often considered the best strategy. Inadequate retention and adherence lead to decreased health outcomes (morbidity, mortality, drug resistances, risk of transmission) and cost effectiveness (increased costs and lower productivity).
\end{abstract}

S. M. Stricker $(\bowtie) \cdot$ S. de Pee $\cdot$ N. Grede $\cdot$ M. W. Bloem HIV/AIDS and Nutrition Policy Division, United Nations World Food Programme, Via C.G.Viola 68, Parco dei Medici, 00148 Rome, Italy

e-mail: sebastian.stricker@wfp.org

S. de Pee

e-mail: saskia.depee@wfp.org

K. A. Fox $\cdot$ R. Baggaley $\cdot$ E. Negussie Department of HIV/AIDS, World Health Organization, Geneva, Switzerland
Keywords Retention - Adherence · HIV/AIDS · Measurement · ART

\section{Introduction}

Retention in care and adherence to antiretroviral treatment (ART) are critical elements of HIV care and are closely associated with optimal individual and public health outcomes and cost effectiveness. Despite the significance of retention and adherence, a broad body of evidence suggests that many clients struggle to adopt the appropriate behaviours recommended by their health-care providers. This translates specifically to increased morbidity and mortality through suboptimal viral suppression, increased risk of drug resistance, and increased risk of HIV transmission; and more generally to higher health-care costs and a loss of or reduction in individual income, potentially translating to lower economic productivity at the country level.

Consequently, there is increasing concern about inadequate retention and adherence, due, in no small part, to the amount of financial and human resources investment required to sustain or expand health-care services. Through global effort, ART coverage increased by more than $2,500 \%$ between 2002 and 2011 in low- and middleincome countries, resulting in treatment coverage of just over 8 million people living with HIV (PLHIV), or $54 \%$ of those eligible are receiving treatment $[1,2]$. Despite the expansion of HIV services, programme data indicates that PLHIV often encounter multiple challenges to accessing available health-care.

This paper reviews the published literature on retention in HIV care and adherence to treatment and aims to clarify terminology used, provide an overview of retention and adherence research, discuss measurement methods, and 
describe the consequences of inadequate retention and adherence.

\section{Understanding the Concepts of Retention and Adherence}

In the last four decades, literature on health care and public health has focused increased attention on the complexities and importance of the patient-health-care provider relationship, also understood as a supply (health-care provider and system)-demand (patient) paradigm [3]. With the steady increase in quality and reach of health services, some experts suggest that efforts to increase client involvement may have a greater impact on health outcomes than any other improvement in specific medical treatments [4].

A review of recent literature reveals a range of terms also used to describe retention in care and adherence to treatment, including: 'compliance', 'concordance', 'persistence', and more simply, 'retention' and 'adherence' [3, 5-8]. Altogether, they aim to describe the conformity of a person's behaviour with that recommended by their healthcare provider(s). However, each word has a specific meaning and implications for its use. 'Compliance' may imply a paternalistic relationship between health-care providers and clients and stigmatize those considered to be not complying with their health-care provider's advice [3, 7, 9]. Alternatively, some advocate for 'concordance', which is meant to more accurately reflect the reciprocal relationship between health-care providers and their clients $[10,11]$. The World Health Organization (WHO) uses 'retention' (in care) and 'adherence' (to treatment) as a preferred terminology, and as such, this article will use the same terms. Simply defined, they are:

- Retention (in care) a client's continued engagement in health services and captures the whole 'continuum of HIV care': from enrolment in care to discharge/death of the client;

- Adherence (to treatment) signifies the extent a client follows a prescribed medication or treatment regimen $[2,12]$.

Based on these definitions, a client can be 'retained in care' (e.g., attending a clinic appointment according to schedule), but not necessarily 'adhere to treatment' (e.g., fail to take medication as prescribed). However, in general, the best outcomes are achieved if a client is retained in care (so that they can be clinically monitored) and adheres to treatment. The above definitions rely on a minimum requirement that $\mathrm{ARVs}$ be available. An inadequate supply of ARVs at the facility or country levels can interrupt treatment, leading to poor adherence, and possibly to poor retention.
'Loss to follow-up' and 'attrition' are terms often used in the literature to represent clients who disengaged from care at any stage of the continuum of care. How loss to follow-up is quantified varies greatly in the field, and in some cases, even within some studies. Lamb et al.'s [13] study of the association of adherence support and services in relation to loss to follow-up and attrition, used two types of data analysis and two different definitions for loss to follow-up_cumulative analysis, in which patients were considered loss to follow-up if they had not visited a clinic within the previous three months 'without documented evidence of death or transfer to another clinic' and those who returned to care were no longer defined as lost to follow-up; and cohort analysis, in which patients were considered lost to follow-up after six and twelve months, regardless of whether or not they eventually returned to ART care. Loss to follow-up can also be more broadly defined, depending on the context, to consider those who choose not to continue with their treatment or encounter other barriers to continued access to treatment. In that respect, loss to follow-up is not an outcome by itself. The varied definitions of loss to follow-up within Lamb's study alone, underscores the challenge of comparing research on retention across the field.

Loss to follow-up and attrition are often difficult to quantify, especially in resource-limited settings where unique client identifiers and vital registers may be lacking. Additionally, most studies focus on selected stages, such as the rate of clients initiating treatment after diagnosis, or the rate of clients still on treatment at a specific period in time instead of the entire continuum of care (Exhibit 1).

\section{Monitoring Client Retention and Adherence}

A growing body of studies examine client retention and adherence rates, but interpretation needs to be handled with caution. At present, there is no consensus on period definitions for retention or what constitutes 'adequate' retention and adherence (e.g., what type or level of client behaviour). Similarly, indicators are not standardized making it difficult to compare across programmes, or differences in retention and adherence over time. In this sense, some studies consider clients to be lost to follow-up as early as one day after missing an appointment, while others begin count as late as 180 days or a year from the last client facility visit. Many programmes report on basis of dichotomous variables, such as in-care versus loss to follow-up or adherent versus nonadherent. There is also a lack of consistency with regard to the inclusion of deceased clients (and thus not following-up) $[14,15]$. Other critical issues include self-transferred clients-when people decide to enrol in care at a new health facility without informing staff at their previous clinics — and 
Exhibit 1 Continuum of care and contingencies for loss to follow-up

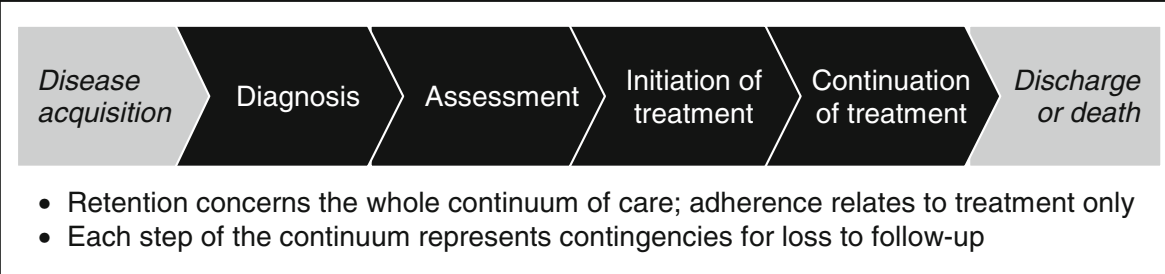

unascertained death where vital registration data is not routinely collected in many resource-limited settings. Due to the high resource demand associated with tracking clientscompounded by the lack of unique client identifiers, especially in resource-limited settings-some studies may not succeed in identifying the true outcome of those who are lost to follow-up, consequently leading to over-reporting of loss to follow-up. Other issues that impair data comparability include the study settings (e.g., formal clinic trials vs. largescale programmes in resource-limited settings) and the measurement method applied (e.g., self-reporting may be associated with an overestimation of retention in care and treatment adherence) [12, 16-18].

Inadequate retention and adherence affects virtually all client-health-service relationships, independent of the type of disease, however, there are differing observations with regard to client predictors of loss to follow-up and nonadherence, such as ethnicity, age, or gender [19-22]. In general, retention and adherence rates appear to be higher for conditions with short course of illness [23, 24]. Also, there is some evidence that indicates better ART adherence in resource-limited settings compared to some resourcerich settings $[25,26]$. Based on a meta-analysis of 569 studies, DiMatteo [27] found an average non-adherence rate of $24.8 \%$ of clients, highest in HIV, arthritis, gastrointestinal disorders, and cancer, and lowest in pulmonary disease, diabetes, and sleep disorders.

Reaching adequate retention in HIV care and ART adherence may be more difficult and may have more complex challenges than for many other diseases. This is due to several factors: (1) HIV is a chronic condition requiring lifelong treatment, (2) HIV-related stigma can be a significant barrier, and (3) complex treatment regimens that can also have undesirable adverse effects and drug interactions. Loss to follow-up and poor adherence undermine individual health outcomes, HIV prevention potential and could have implications on the cost effectiveness of HIV treatment.

The following is a brief overview of recent literature on retention and adherence, organized along the different steps of the continuum of care:

- Diagnosis - a major challenge in tackling the HIV epidemic is the failure to promptly diagnose HIV infections. In 2010, a median number of 55 tests per 1,000 adult population were conducted (up from 47 in
2009), with the highest number of tests occurring in Sub-Saharan Africa $(82$ per 1,000) and the lowest in East, South and South-East Asia $\left(22\right.$ per 1,000) [2]. ${ }^{1}$ Despite the increased scale up of HIV tests and testing facilities, the level of testing remains insufficient overall. PLHIV unaware of their status range from approximately $69 \%$ in Congo and $31 \%$ in Kenya [2] to $33 \%$ in Europe [28] and $20 \%$ in the US [29]. Failure to diagnose HIV infection is usually highest among clients who are not perceived to be at risk of HIV infection, or are marginalized (e.g., injecting drug users, men who have sex with men, immigrants) [30].

- Assessment and initiation of care and treatment-once clients are diagnosed with HIV, it is crucial to link and enrol them to care and treatment services. Unfortunately, several studies suggest that only a fraction of people diagnosed with HIV actually start treatment. In their systematic review looking at retention in HIV care between HIV testing and counselling (HTC) and initiation of ART, Rosen and Fox [31] found that $59 \%$ of clients are lost between diagnosis and assessment (receipt of CD4 count or clinical staging), $46 \%$ of clients are lost between assessment and eligibility for ART, and $68 \%$ between eligibility and ART initiation. Similarly, data from the Viet Nam Authority for HIV/ AIDS Control lists only $31.6 \%$ of (diagnosed) PLHIV as enrolled in care (pre-ART and ART) [2]. In contrast, Gardner et al. [32] estimate that $\sim 75 \%$ of newly diagnosed PLHIV in the United States are linked to HIV care within 6-12 months after diagnosis, and 80-90\% linked within 3-5 years. Measured by the number of days from diagnosis to the start of ART, delays for clients in South Africa were estimated at 78 and 34 days in Free State Province [33] and Cape Town, South Africa [34], respectively, and about 40 days in Cambodia [35].

- Retention in care and adherence to treatment-once ART is initiated, clients need to be retained and they need to adhere to treatment. A systematic review of 33 sources describing 39 cohorts and 226,307 clients in sub-Saharan Africa calculated that a median of $86.1 \%$

\footnotetext{
${ }^{1}$ Please note that these numbers relate to total number of tests, as opposed to individuals tested. The number of people testing is likely lower as some may take multiple tests over the course of a year.
} 
of clients remained in the programme at 6 months, $80.2 \%$ at 12 months, $70.0 \%$ at 24 months and $64.6 \%$ at 36 months. Of those reported as lost to follow-up, an average of $41 \%$ of clients were identified to be deceased [36]. In 2011, data from 149 low- and middle-income countries indicated an average retention rate of $81 \%$ at 12 months, $75 \%$ at 24 months, and $67 \%$ at 60 months from 92, 73, and 46 countries, respectively [2]. It is noteworthy that loss to follow-up in both studies was highest in the first 12 months, presumably due to early mortality on ART.

In terms of adherence to treatment, i.e., how reliably clients follow the prescribed medication regimen, Ortego et al. [26] found that an average rate of $62 \%$ of clients reported $\geq 90 \%$ adherence. In this meta-analysis, good adherence was more likely in studies with "higher proportions of men who have sex with men (MSM) and lower proportions of injection drug users (IDU), with participants in an earlier stage of HIV infection, [and] in countries characterized by lower Human Development Index (HDI) scores" [26]. Similar results were reported from a metaanalysis conducted by Mills et al. [25], who found that a pooled estimate of $55 \%$ of the populations in North America and $77 \%$ in twelve sub-Saharan African countries achieved adequate levels of adherence. Gardner et al. [32] reports that $\sim 60 \%$ of US clients in need of ART and $\sim 80 \%$ of those on ART are adherent/achieve viral suppression.

\section{Measuring Retention and Adherence}

Health intervention effectiveness is highly dependent on understanding the role of patient/client behaviour and appropriately measuring the levels of both retention and treatment adherence. Health-care providers need to 'measure what they manage', in other words, monitor clients' behaviour during care and treatment to optimize outcomes. It is also crucial to recognize that if the effectiveness of an intervention is evaluated based on outcomes with differing measurement approaches of client retention and adherence, this impairs comparability and consequently the identification of appropriate responses in a given setting [37, 38].

While it is clear that measuring retention to care and adherence to treatment is critical, doing so accurately is complex and, at present, no globally agreed on 'goldstandard' of measurement exists. Measuring adherence objectively in routine-care settings is often a challenge because of potential issues related to approaches originating from feasibility, cost, and health-care providers' time. Programmes and research use different methods to measure adherence to treatment, each with specific advantages and disadvantages. Approaches are usually divided into direct and indirect methods [9, 39]: 'direct' refers to monitoring of the implementation of the intervention or its immediate outcomes; and 'indirect' refers to the use of proxy indicators that are associated with adherence. Consequently, direct approaches are often regarded as more accurate, while indirect methods may be more feasible to implement (and thus also more commonly used) [16].

Direct approaches to measuring adherence include: directly-observed treatment and biochemical analysis of compounds in bodily fluids. An example for direct observation would be handing out and taking drugs in the presence of health care staff. Alternatively, biochemical analysis of compounds in bodily fluids measures drug adherence through detection of either the medication itself (or its metabolites) or non-toxic markers that have been added to the regimen.

Indirect approaches to measuring ART adherence include viral load monitoring, client self-reporting (i.e., client interviews, questionnaires and diaries), pill-counting, prescription claims/pill refill, electronic monitoring devices, client clinic attendance, and provider reporting. Client self-reports are widely employed, however, they are inherently subjective, prone to recall bias, and often overestimate adherence if patients shy from reporting any lapses in adherence due to fear of criticism from their care providers [12, 16-18]. Another, widely employed indirect measurement method is pill-counting, which is usually performed during clinical appointments and, if the date of the last refill is known, allows calculation of drug adherence. However, pill counting at the clinic may lead to 'pill dumping' if a client knows that this may be routinely assessed. Unannounced home visits for pill counting have also been employed as part of adherence monitoring in clinical trial settings. The downside of this method is that it is time consuming and clients may perceive it as 'policing', thus posing a challenge to client-provider trust. Additionally, it can be difficult to ascertain whether the client has followed the prescribed frequency and/or dosage through pill counting alone. Prescription claims/pill refills follow a similar logic, using the timing and/or frequency of these as indicators, and is potentially immune to social desirability or reporting bias. A comparison of different adherence monitoring approaches indicates that pharmacy records more accurately correlate with ART adherence compared to self-reporting. Electronic monitoring devices are a relatively new but expensive measurement method that records the date and time the drug container is openedproviding detailed information on patterns of medication taking. This method is not commonly used in routine care settings due to high costs associated with it. Client intervention outcomes may also be used as indicators of adherence, as clinical (e.g., return to health) and biological 
outcomes (e.g., suppressed viral load), in certain cases, can only be achieved with adequate adherence. Client clinic attendance monitors timing and frequency of clinic visits, and is the most common measurement method to assess retention in care. However, most programmes do not routinely monitor retention in care, and routine programme cohort analysis needs to be strengthened in several settings.

The selection of the most appropriate measurement approach needs to be at least reliable (i.e., consistent across time and settings) and valid (i.e., measures what it purports to measure) [40, 41]. It also needs to take into account: goal of measurement (i.e., research/documentation vs. routine care), type of intervention (e.g., drug intervention vs. lifestyle changes), length of intervention, number of and access to clients, available resources, and ethical and legal considerations. In practice, combining more than one measurement method, e.g., subjective client self-reporting with more objective pharmacy-based pill refills, is often considered the most feasible approach in resource-limited settings [12, 40, 42], or reflecting the impact of the intervention, as in viral load suppression. With regard to the latter, for those taking ART, viral suppression is usually an indication that they are adhering effectively to prescribed medicines. The converse may not always be true as someone may be adhering to their ART but if they had been given a regimen which was effective against their viral strain this would not result in viral suppression.

In principle, all of the above measurement methods may be applied in HIV care. However, from a programmatic perspective, especially in resource-limited settings, direct approaches are usually considered less feasible given the comparatively high amount of resources associated with it (especially for life-long treatment) [17, 43-45]. For measuring the impact/effectiveness of a programme measuring viral suppression is key (Exhibit 2).

\section{Retention and Adherence in the Response to HIV}

Retention and adherence play a critical role in the response to HIV, spanning across the whole continuum of care: from diagnosis and assessment to the initiation and continuation of treatment. Retention in care and adherence to antiretroviral therapy (ART) need to be maintained for lifepresenting significant challenges for clients and health-care providers alike as the epidemic evolves from one requiring an emergency response to one necessitating long-term comprehensive care.

Several factors underscore the importance of retention and adherence in the response to HIV. UNAIDS and WHO estimate that the rate of new HIV infections are decreasing-by $19 \%$ between 2002 and 2011-indicating that prevention efforts have been successful in many settings (although 2.5 million new HIV infections occurred in 2011) $[1,2]$. This success is due in part to the significant expansion of access to HIV related-health services in lowand middle-income countries where ART coverage increased more than 2,500\% during 2002-2011 [1, 2]. For the 34 million people presently living with HIV, most will reach $\mathrm{CD} 4$ cell count levels requiring treatment initiation within this decade (as of 2011, approximately 8 million PLHIV were receiving ART, with 6.8 million still eligible) [1]. In addition, the effectiveness of ARVs is improving, substantially reducing morbidity and mortality [46-49], and simpler, less toxic ARV regimens are gradually being introduced in low-income countries.

\section{Consequences of Inadequate Retention in Care and Adherence to ART}

Inadequate retention in care and adherence to treatment are widely associated with significant negative implicationssuch as reduced programme effectiveness and increased morbidity and mortality - for the client and population as a whole [12]. Amongst other things, the development of drug resistance is related to suboptimal adherence [50], reducing treatment effectiveness. Suboptimal adherence may also bear population-level implications, specifically through increased risks of transmission, including transmission of drug-resistant strains (Exhibit 3).

As a consequence of suboptimal health outcomes, insufficient retention and adherence also negatively affect cost effectiveness of health interventions (i.e., increases direct health-care costs and indirect loss of income/productivity). In the case of health-care costs, these are largely dependent on the health-care financing systems of a country. For loss of income/productivity, the implications may be greater if the consequences of reduced productivity on an individual level and its effects on other, healthy, individuals are taken into consideration (i.e., reduced productivity of one individual may impair productivity for other individuals).

Retention in care and adherence to ART have significant implications on health outcomes and cost effectiveness of HIV interventions for both PLHIV and society as a whole. Failure to diagnose HIV and/or delayed care and treatment initiation ('late presentation') are associated with higher rates of on-going HIV transmission, greater risk of opportunistic infections, reduced treatment effectiveness, and significantly increased short-term mortality in 'late presenters' compared with clients who are diagnosed and start treatment earlier [51-53]. Several studies have identified poor treatment adherence, second only to CD4 cell count, as a strong predictor of HIV progression to AIDS and ultimately death [54-56]. 


\section{Advantages}

- Allows holistic monitoring of actual

Observation of intervention execution

Direct

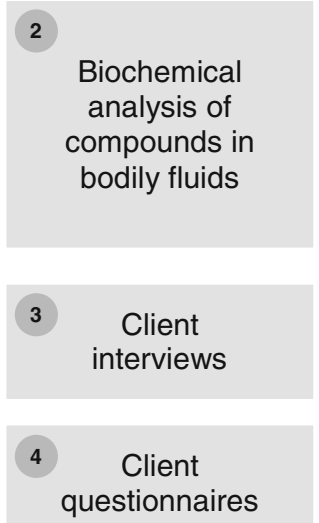

5

Client diaries

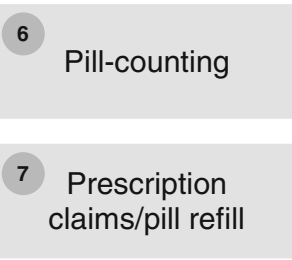

Indirect
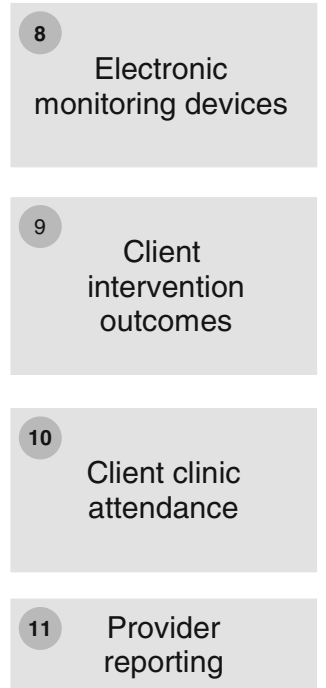
intervention (e.g., timing, 'quality' of adherence)

- Avoids client recall (e.g., reporting of socially expected behaviour)

\section{- Avoids client and provider recall or bias (e.g., reporting of socially expected behaviour) issues}

- Standardized, quantified measurement outcomes

- Flexibility on data gathering depending on client responses

- Low resource requirements

- Standardized, partially quantitative measurement outcomes

- Low resource requirements

- Avoids client recall issues

- Standardized, quantified measurement outcomes

- Objective

- Low resource requirements

- Avoids client bias or recall issues

- Standardized, quantified measurement outcomes

- Analysis of timing of drug intake

- Avoids client and provider bias or recall issues

- Objective

- Low resource requirements

- Avoids client bias or recall issues

- Low resource requirements

\section{Disadvantages}

- Potential strong Hawthorne effect

- Resource-intensive, thus limited applicability (e.g., clinic settings)

- Results influenced by metabolism and/or other external factors

- Resource-intensive

- Limited breadth of information (e.g., reasons for non-adherence)

- Potential strong Hawthorne effect

- Relatively resource intensive

- Potential client/prescriber bias and recall issues

- Potential client bias and recall issues

- Requires client literacy

- Potential client bias issues

- Low levels of standardization

- Potential high effort for client

- Potential data distortion (e.g., pill dumping, refill from other channels)

- Limited breadth of information

- Potential data distortion (e.g., pill dumping, refill from other channels)

- Limited breadth of information

- Potential data distortion (e.g., pill dumping, refill from other channels)

- Resource-intensive

- Results may be influenced by other factors (e.g., client diet)

- Resource-intensive

- Limited breadth of information

- Potential weak relationship with adherence to treatment

- Client transfers to other facilities

- Limited breadth of information

- Limited prescriber insight in actual client behaviour

Exhibit 2 Retention and adherence measurement methods and respective advantages and disadvantages 


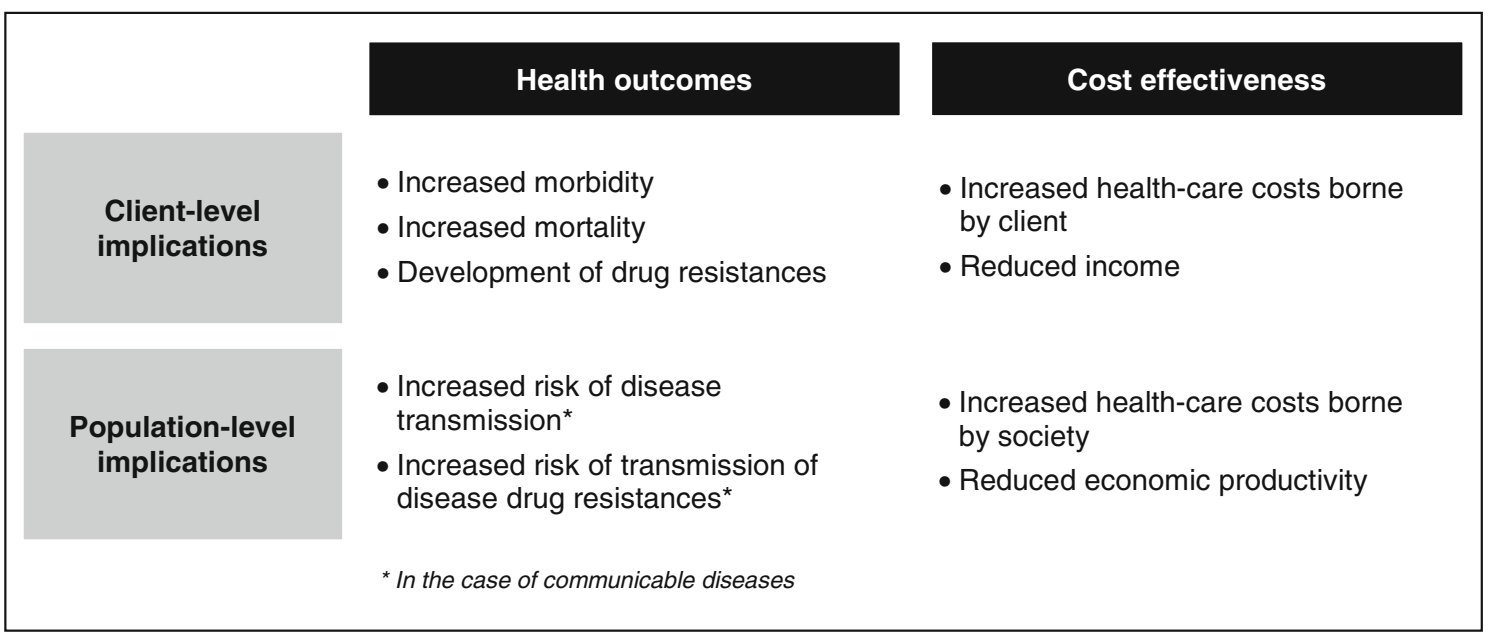

Exhibit 3 Potential consequences of inadequate retention in care and adherence to treatment

Relatively small differences in medication intake can trigger major differences in viral suppression. Using different methods of measuring data, several studies analysed the relationship between adherence and viral load and/or mortality. Grossberg et al. [57] found that a $10 \%$ increased adherence rate was associated with viral load decreases of $0.12 \log \mathrm{c} / \mathrm{mL}$ (adherence measured through drug refills). Similarly, Paterson et al. [58] found that virologic failure occurred in only $22 \%$ of clients with $95 \%$ or greater adherence compared to clients with 80-94.9\% adherence $(61 \%)$ and those with under $80 \%$ adherence $(80 \%)$ (adherence was measured through electronic intervention monitors). In terms of mortality, adherence has also been shown to be a critical determinant of client survival. A population-based analysis of 1,282 ART-naive HIVpositive individuals in British Columbia found that clients who used antiretroviral drugs intermittently (adherence level $<75 \%$ ) were 2.97 times more likely to die (after adjustment for other prognostic factors, measuring adherence through drug refills) [56]. Similarly, Nachega et al. [59] analysed survival among 6,288 HIV-1-infected South African adults enrolled in a private-sector AIDS management program and found that adherence levels below $80 \%$ were associated with a 3.23 higher mortality. When drug adherence was divided into quintiles (20\% each), each quintile had lower survival rate than the next higher one (adherence measured through prescription claims). Using clinic visits as the predictor, Giordano et al. [60] also come to comparable findings: based on a total of 2,619 men followed for a mean of more than 4 years, clients with visits in three quarters, two quarters, and one quarter in the first year had a 1.42, 1.67 and 1.95 higher mortality respectively compared with clients with visits in all four quarters.

In addition to increases in morbidity and mortality, inadequate adherence reduces treatment effectiveness due to drug resistances, and also limits ARV options. There has been extensive discussion on the relationship between client adherence levels and the prevalence of drug resistance [61, 62]. Earlier studies indicate a bell-shaped curve (i.e., suppression of drug resistances is best at medium-high levels, less at low- and near-perfect adherence). More recently, studies have shown that each regimen class may have different adherence-resistance relationships [63, 64]. Gardner et al. [65] have found that 'differential' adherence-defined as any difference in self-reported adherence levels to individual antiretroviral drugs at the same time (when on a multiple-pill drug regimen) - is associated with increased antiretroviral resistance. HIV treatment guidelines from both WHO and the US Department of Health and Human Services stress the importance of adherence to suppress drug resistances and improve health outcomes $[66,67]$.

While inadequate retention and adherence negatively impact individual health outcomes, evidence suggests that it also has population-level implications. Transmission of drug resistances following poor treatment adherence can lead to suboptimal response to first-line therapy in newly diagnosed PLHIV [68]. However, the prevalence of transmitted drug resistances is increasing, but at lower rates than some had feared based on the significant expansion of ART coverage [69]. Treatment adherence has also a critical role in prevention of HIV transmission. Knowledge of HIV status allows PLHIV to adopt risk-reduction behaviours. Evidence from the randomised control trial HPTN 052 confirmed findings from previous cohort studies that by reducing virus levels in bodily fluids, ART has the potential to significantly reduce HIV transmission [70-72]. However, in order to harness the prevention benefits of ART, clients need to adhere to medication.

Retention and adherence are also critical determinants of programme cost effectiveness: due to higher morbidity, client health-care needs increase, and potential drug 
resistance makes more expensive second-/third-line treatment necessary. In a study on cost implications from suboptimal medication adherence, Nachega et al. [73] followed 6,833 HIV-infected adults in South Africa between August 2000 and April 2006, monitoring ART, hospitalizations, consultations, investigations and nonART medications. Clients were grouped in four adherence quartiles, with total mean costs ranging from $\$ 313$ for highest adherence to $\$ 376$ for lowest adherence per month (adherence was measured through prescription claims). Although ART costs alone were higher in clients with better adherence, the total mean costs were offset by significantly reduced hospitalization costs. Comparable results were found in two earlier studies: Knobel et al. [74], examining the influence of treatment adherence on hospital admissions in patients on HAART in Barcelona, calculated mean hospital days per admission of 3.73 for adherent clients ( $>90 \%$ adherence), as opposed to 14.75 days for non-adherent clients (adherence was measured through client interviews and pill counts); and Paterson et al. [58], looking at combination ART with protease inhibitors in patients in Pittsburgh, found that clients with above $95 \%$ adherence had fewer hospital days (2.6 days per 1,000 days of follow-up) and developed fewer opportunist infections than less adherent clients (12.9 days per 1,000 days of follow-up) (adherence was measured through electronic interventions monitors).

Drug resistances resulting in the need to start more costly treatment regimens earlier is emphasized by the significant price differences between first- and second-line ARV regimens. In 2010, the most commonly used adult first-line ART regimens in low- and middle-income countries cost on average $\$ 124$ per person per year, compared with $\$ 554$ for second-line drugs (price difference of $347 \%$ ) [2]. In this regard, Long et al. [75] calculated that total second-line outpatient health-care costs are 2.4 times higher than firstline. Predictably, the biggest increase came from higher costs of second-line drugs, with limited additional expenditure for laboratory services and clinic visits. Because of cost implications, Long et al. stress the importance of limiting or delaying the number of clients requiring second-line therapy. Furthermore, second-line treatment is often not widely available in resource-limited settings.

\section{Treatment 2.0}

WHO and UNAIDS have also put increasing emphasis on the need to support retention in care and adherence to ART for PLHIV. The Treatment 2.0 Framework, an initiative launched in 2010 seeking to address the financial and technical challenges in achieving universal access, defines five priority areas for future efforts in HIV-each is interlinked with retention in care and adherence to treatment [76]:

1. 'Optimize drug regimens' calls for "effective, affordable, one pill, once daily potent ARV regimens with minimal toxicities or drug interactions". This can be expected to have significant positive impact on adherence, as several studies validate the relationship between ease of drug use, such as pill burden, and actual intake [77-80].

2. 'Provide access to point-of-care and other simplified diagnostics and monitoring tools' addresses the insufficient level of HIV testing and counselling and CD4 testing and monitoring leading to late start of HIV treatment, which in turn is associated with increased morbidity and mortality [81, 82]. A recent study from Mozambique indicates that availability of point of care CD4 testing enables clinics to stage clients quickly onsite, reducing risks for pre-treatment loss to follow-up among those who are eligible for ART at time of assessment [83].

3. 'Reduce costs' emphasizes that it is necessary to ensure the most effective use of limited available resources (e.g., through pooled-drug procurement and diagnostics) [84]. While such activities would likely not directly affect retention and adherence, it is obvious that retention and adherence can maximise programme efficiency.

4. 'Adapt delivery systems' emphasizes the need for a decentralized HIV response, integrated with other health services. Recent studies from Zambia suggest that the provision of ART integrated with maternal and child health services significantly increased treatment initiation [85, 86]. Similarly, a study from South Africa indicates that integrating ART with tuberculosis care improves treatment coverage among co-infected clients [87]. Factors such as transport costs and waiting time have been identified as major barriers to retention and adherence [88, 89], indicating that proximity as well as convenience of service delivery are critical.

5. 'Mobilize communities' calls for a better integration and linkages of the 'informal' community sector in the response to HIV. Engaging communities in the creation, development, and implementation of programmes and services can provide a more enabling environment that supports quality of care, retention in care, and treatment adherence for their populations. While the health-care sector can strive to improve the availability and quality of HIV services, many barriers to retention in care and adherence to treatment exist outside their purview (e.g., disability, stigma, poverty). Unfortunately, the health-care sector is often not well positioned to address many of these issues. However, 
by actively involving communities-through coordination with and strengthening of health-care and other key sectors-significant improvements can occur at both the individual- and community-levels to overcome these barriers, resulting in increased access to HIV services, stigma reduction, retention in care, and treatment adherence [90-92].

The importance of retention and adherence is also reflected in the 2011 General Assembly Political Declaration on HIV/AIDS, which emphasizes the need to "address factors that limit treatment uptake and contribute...to poor adherence" and calls for the "mobilization and capacitybuilding of communities to support treatment scale-up and patient retention" as well as "programmes that support improved treatment adherence" [93]. A focus on the client side was also underscored by UNAIDS in their 2011-2015 strategy, which stated that "the demand side of treatmentthe factors that make people enrol for treatment and adhere to it-has not received enough attention" [94].

\section{Conclusions and Areas for Further Research}

Retention in care and adherence to treatment are critical for health outcomes and cost effectiveness of health interventions. Disease-generic as well as HIV-specific data suggest, for a variety of reasons, that clients often face challenges following the behaviour recommended by their health-care providers. This holds true for both resource-rich and resource-limited settings.

Major challenges to the response to HIV include: prompt diagnosis of HIV infection, poor linkage to care, delayed treatment initiation, and insufficient adherence to treatment. Historically, attention was focused primarily on the latter stage (continuation of treatment); however, it becomes apparent when examining the continuum of HIV care that most client loss to follow-up happens following testing and before start of ART. On both the client- and population-levels, failure to link clients to care following testing and inadequate retention and adherence to ART, once in care, result in significant increases in morbidity and mortality and are associated with on-going transmission of HIV infection. Additionally, suboptimal viral suppression may result a higher risk of developing drug resistances, as well as the transmission of drug resistance. From a costeffectiveness perspective, inadequate retention and adherence may lead to an increase of health-care costs.

Current suboptimal retention in care and adherence to ART threaten the benefits of the on-going scale up of HIV care and treatment, and may result in a failure to capitalise on the treatment and prevention benefits of ART. Therefore, it is crucial that retention and adherence are put at the heart of the HIV response: to better understand barriers to retention and adherence; to identify, design, and implement programmes to overcome these barriers; and to monitor and evaluate their impact and acceptability.

\section{References}

1. Joint United Nations Programme on HIV/AIDS (UNAIDS). Together We Will End AIDS. Geneva: UNAIDS; 2012.

2. World Health Organization (WHO), United Nations Children's Fund (UNICEF), Joint United Nations Programme on HIV/AIDS (UNAIDS). Global HIV/AIDS Response: Epidemic Update and Health Sectors Progress Towards Universal Access. Progress Report 2011. Geneva: WHO; 2011.

3. Vermeire E, Hearnshaw H, Van Royen P, Denekens J. Patient adherence to treatment: three decades of research. A comprehensive review. J Clin Pharm Ther. 2001;25(5):331-42.

4. Haynes RB, McDonald H, Garg AX. Montegue P. Cochrane Database Syst Rev: Interventions for helping patients to follow prescriptions for medications; 2002 (2):CD000011. [NOTE: updated 2005;(4):CD000011.].

5. DeMaria AN. Adherence, compliance, concordance, or the lack thereof. J Am Coll Cardiol. 2012;59(12):1120-1.

6. Aronson JK. Compliance, concordance, adherence. Br J Clin Pharmacol. 2007;63(4):383-4.

7. Bell SJ, Airaksinen MS, Lyles A, Chen TF, Aslani P. Concordance is not synonymous with compliance or adherence. Br J Clin Pharmacol. 2007;64(5):710-1.

8. Steiner EM, Earnest MA. The language of medication-taking. Ann Intern Med. 2000;132:926-30.

9. Osterberg L, Blaschke T. Adherence to medication. N Engl J Med. 2005;353(5):487-97.

10. Cramer JA, Roy A, Burrell A, et al. Medication compliance and persistence: terminology and definitions. Value Health. 2008;11(1):44-7.

11. Bissell P, May CR, Noyce PR. From compliance to concordance: barriers to accomplishing a re-framed model of health care interactions. Soc Sci Med. 2004;58(4):851-62.

12. World Health Organization. Adherence to long-term therapies: evidence for action. Geneva: WHO; 2003.

13. Lamb MR, El-Sadr WM, Geng E, Nash D. Association of adherence support and outreach services with total attrition, loss to follow-up, and death among ART patients in sub-Saharan Africa. PLoS One. 2012;7(6):e38443.

14. Chi BH, Cantrell RA, Mwango A, et al. An empirical approach to defining loss to follow-up among patients enrolled in antiretroviral treatment programs. Am J Epidemiol. 2010;171(8):924-31.

15. Chi BH, Yiannoutsos CT, Westfall AO, et al. Universal definition of loss to follow-up in HIV treatment programs: a statistical analysis of 111 facilities in Africa, Asia, and Latin America. PLoS Med. 2011;8(10):e1001111.

16. Fairman K, Motheral B. Evaluating medication adherence: which measure is right for your program? J Manage Care Pharm. 2000;6:499-504.

17. Berg KM, Arnsten JH. Practical and conceptual challenges in measuring antiretroviral adherence. J Acquir Immune Defic Syndr. 2006;43(Suppl 1):S79-87.

18. Miller LG, Hays RD. Measuring adherence to antiretroviral medications in clinical trials. HIV Clin Trials. 2000;1(1):36-46.

19. Mannheimer S, Friedland G, Matts J, Child C, Chesney M. The consistency of adherence to antiretroviral therapy predicts biologic outcomes for human immunodeficiency virus-infected persons in clinical trials. Clin Infect Dis. 2002;34(8):1115-21. 
20. Balkrishnan R. Predictors of medication adherence in the elderly. Clin Ther. 1998;20(4):764-71.

21. Holzemer WL, Corless IB, Nokes KM, et al. Predictors of selfreported adherence in persons living with HIV disease. AIDS Patient Care STDS. 1999;13(3):185-97.

22. Stone VE, Hogan JW, Schuman P, et al. Antiretroviral regimen complexity, self-reported adherence, and HIV patients' understanding of their regimens: survey of women in the her study. J Acquir Immune Defic Syndr. 2001;28(2):124-31.

23. Jackevicius CA, Mamdani M, Tu JV. Adherence with statin therapy in elderly patients with and without acute coronary syndromes. JAMA. 2002;288(4):462-7.

24. Cramer J, Rosenheck R, Kirk G, Krol W, Krystal J, VA Naltrexone Study Group 425. Medication compliance feedback and monitoring in a clinical trial: predictors and outcomes. Value Health. 2003;6(5):566-73.

25. Mills EJ, Nachega JB, Buchan I, et al. Adherence to antiretroviral therapy in sub-Saharan Africa and North America: a meta-analysis. JAMA. 2006;296(6):679-90.

26. Ortego C, Huedo-Medina TB, Llorca J, et al. Adherence to highly active antiretroviral therapy (HAART): a meta-analysis. AIDS Behav. 2011;15(7):1381-96.

27. DiMatteo MR. Variations in patients' adherence to medical recommendations: a quantitative review of 50 years of research. Med Care. 2004;42(3):200-9.

28. Coenen T, Lundgren J, Lazarus JV, Matic S. Optimal HIV testing and earlier care: the way forward in Europe. HIV Med. 2008; 9(Suppl 2):1-5.

29. US Centers for Disease Control and Prevention (CDC). HIV surveillance-United States, 1981-2008. Morb Mortal Wkly Rep. 2011;60(21):689-93.

30. Girardi E, Sabin CA, Monforte AD. Late diagnosis of HIV infection: epidemiological features, consequences and strategies to encourage earlier testing. J Acquir Immune Defic Syndr. 2007;46(Suppl 1):S3-8.

31. Rosen S, Fox MP. Retention in HIV care between testing and treatment in sub-Saharan Africa: a systematic review. PLoS Med. 2011;8(7):e1001056

32. Gardner EM, McLees MP, Steiner JF, del Rio C, Burman WJ. The spectrum of engagement in HIV care and its relevance to test-and-treat strategies for prevention of HIV infection. Clin Infect Dis. 2011;52(6):793-800.

33. Ingle SM, May M, Uebel $\mathrm{K}$, et al. Outcomes in patients waiting for antiretroviral treatment in the Free State Province, South Africa: prospective linkage study. AIDS. 2010;24(17):2717-25.

34. Lawn SD, Myer L, Harling G, Orrell C, Bekker LG, Wood R. Determinants of mortality and nondeath losses from an antiretroviral treatment service in South Africa: implications for program evaluation. Clin Infect Dis. 2006;43(6):770-6.

35. Thai S, Koole O, Un P, et al. Five-year experience with scalingup access to antiretroviral treatment in an HIV care programme in Cambodia. Trop Med Int Health. 2009;14(9):1048-58.

36. Fox MP, Rosen S. Patient retention in antiretroviral therapy programs up to three years on treatment in sub-Saharan Africa, 2007-2009: systematic review. Trop Med Int Health. 2010; 15(Suppl 1):1-15.

37. Urquhart J. Defining the margins for errors in patient compliance with prescribed drug regimens. Pharmacoepidemiol Drug Saf. 2000;9(7):565-8.

38. Paes AH, Bakker A, Soe-Agnie CJ. Measurement of patient compliance. Pharm World Sci. 1998;20(2):73-7.

39. Farmer KC. Methods for measuring and monitoring medication regimen adherence in clinical trials and clinical practice. Clin Ther. 1999;21(6):1074-90.

40. Vitolins MZ, Rand CS, Rapp SR, Ribisl PM, Sevick MA. Measuring adherence to behavioral and medical interventions. Control Clin Trials. 2000;21(Suppl 5):S188-94.
41. Nunnally JC, Berstein I. Psychometric theory. 3rd ed. New York: McGraw-Hill; 1994.

42. McMahon JH, Jordan MR, Kelley K, et al. Pharmacy adherence measures to assess adherence to antiretroviral therapy: review of the literature and implications for treatment monitoring. Clin Infect Dis. 2011;52(4):493-506.

43. Chalker JC, Andualem T, Gitau LN, et al. Measuring adherence to antiretroviral treatment in resource-poor settings: the feasibility of collecting routine data for key indicators. BMC Health Serv Res. 2010;19(10):43.

44. Ross-Degnan D, Pierre-Jacques M, Zhang F, et al. Measuring adherence to antiretroviral treatment in resource-poor settings: the clinical validity of key indicators. BMC Health Serv Res. 2010;19(10):42.

45. Steel G, Nwokike J, Joshi MP. Development of a Multi-Method Tool to Measure ART Adherence in Resource-Constrained Settings: The South Africa Experience. Submitted to the USAID by RPM plus. Arlington, Va: Management Sciences for Health; 2007.

46. Herbst AJ, Cooke GS, Bärnighausen T, KanyKany A, Tanser F, Newell ML. Adult mortality and antiretroviral treatment roll-out in rural KwaZulu-Natal, South Africa. Bull World Health Organ. 2009;87(10):754-62.

47. Hammer SM, Squires KE, Hughes MD, et al. A controlled trial of two nucleoside analogues plus indinavir in persons with human immunodeficiency virus infection and CD4 cell counts of 200 per cubic millimeter or less. AIDS Clinical Trials Group 320 Study Team. N Engl J Med. 1997;337(11):725-33.

48. Michaels SH, Clark R, Kissinger P. Declining morbidity and mortality among patients with advanced human immunodeficiency virus infection. N Engl J Med. 1998;339(6):405-6.

49. Chan KC, Wong KH, Lee SS. Universal decline in mortality in patients with advanced HIV-1 disease in various demographic subpopulations after the introduction of HAART in Hong Kong, from 1993 to 2002. HIV Med. 2006;7(3):186-92.

50. World Health Organization (WHO). WHO Global Strategy for Containment of Antimicrobial Resistance. Geneva: WHO; 2001.

51. Phillips A, Pezzotti P. CASCADE Collaboration. Short-term risk of AIDS according to current CD4 cell count and viral load in antiretroviral drug-naive individuals and those treated in the monotherapy era. AIDS. 2004;18(1):51-8.

52. Sabin CA, Smith CJ, Gumley H, et al. Late presenters in the era of highly active antiretroviral therapy: uptake of and responses to antiretroviral therapy. AIDS. 2004;18(16):2145-51.

53. Hocking JS, Rodger AJ, Rhodes DG, Crofts N. Late presentation of HIV infection associated with prolonged survival following AIDS diagnosis—characteristics of individuals. Int J STD AIDS. 2000;11(8):503-8.

54. Bangsberg DR, Perry S, Charlebois ED, et al. Non-adherence to highly active antiretroviral therapy predicts progression to AIDS. AIDS. 2001;15(9):1181-3.

55. de Olalla PG, Knobel H, Carmona A, Guelar A, López-Colomés JL, Caylà JA. Impact of adherence and highly active antiretroviral therapy on survival in HIV-infected patients. J Acquir Immune Defic Syndr. 2002;30(1):105-10.

56. Hoggs RS, Heath K, Bangsberg D, et al. Intermittent use of triplecombination therapy is predictive of mortality at baseline and after 1 year of follow-up. AIDS. 2002;16(7):1051-8.

57. Grossberg R, Zhang Y, Gross R. A time-to-prescription-refill measure of antiretroviral adherence predicted changes in viral load in HIV. J Clin Epidemiol. 2004;57(10):1107-10.

58. Paterson DL, Swindells S, Mohr J, et al. Adherence to protease inhibitor therapy and outcomes in patients with HIV infection. Ann Intern Med. 2000;133(1):21-30.

59. Nachega JB, Hislop M, Dowdy DW, et al. Adherence to highly active antiretroviral therapy assessed by pharmacy claims 
predicts survival in HIV-infected South African adults. J Acquir Immune Defic Syndr. 2006;43(1):78-84.

60. Giordano TP, Gifford AL, White AC Jr, et al. Retention in care: a challenge to survival with HIV infection. Clin Infect Dis. 2007; 44(11):1493-9.

61. Bangsberg DR, Moss AR, Deeks SG. Paradoxes of adherence and drug resistance to HIV antiretroviral therapy. J Antimicrob Chemother. 2004;53(5):696-9.

62. Green DA. There are no paradoxes of adherence and drug resistance to HIV antiretroviral therapy. J Antimicrob Chemother. 2004;54(3):694.

63. Bangsberg DR, Kroetz DL, Deeks SG. Adherence-resistance relationships to combination HIV antiretroviral therapy. Curr HIV/AIDS Rep. 2007;4(2):65-72.

64. Gardner EM, Burman WJ, Steiner JF, Anderson PL, Bangsberg DR. Antiretroviral medication adherence and the development of class-specific antiretroviral resistance. AIDS. 2009;23(9):1035-46.

65. Gardner EM, Sharma S, Peng G, et al. Differential adherence to combination antiretroviral therapy is associated with virological failure with resistance. AIDS. 2008;22(1):75-82.

66. World Health Organization (WHO). Antiretroviral therapy for HIV infection in adults and adolescents: recommendations for a public health approach. 2010 Revision. Geneva: WHO; 2010. p. 2010.

67. Panel on Antiretroviral Guidelines for Adults and Adolescents. Guidelines for the Use of Antiretroviral Agents in HIV-1-Infected Adults and Adolescents. Washington, DC: US Department of Health and Human Services; 2012.

68. Tang JW, Pillay D. Transmission of HIV-1 drug resistance. J Clin Virol. 2004;30(1):1-10.

69. World Health Organization (WHO). WHO HIV Drug Resistance Report 2012. Geneva: WHO; 2012.

70. Cohen MS, Chen YQ, McCauley M, et al. Prevention of HIV-1 infection with early antiretroviral therapy. $N$ Engl J Med. 2011;365(6):493-505.

71. Attia S, Egger M, Müller M, Zwahlen M, Low N. Sexual transmission of HIV according to viral load and antiretroviral therapy: systematic review and meta-analysis. AIDS. 2009;23(11):1397-404.

72. Donnell D, Baeten JM, Kiarie J, et al. Heterosexual HIV-1 transmission after initiation of antiretroviral therapy: a prospective cohort analysis. Lancet. 2010;375(9731):2092-8.

73. Nachega JB, Leisegang R, Bishai D, et al. Association of antiretroviral therapy adherence and health care cost. Ann Intern Med. 2010;152(1):18-25.

74. Knobel H, Carmona A, Guelar A, Gonzolez A, Hernecki J, Diez A. Adherence in patients treated with HAART: influence in hospital admissions. Durban: International AIDS Conference; 2000 [abstract MoPeD2556].

75. Long L, Fox M, Sanne I, Rosen S. The high cost of second-line antiretroviral therapy for HIV/AIDS in South Africa. AIDS. 2010;24(6):915-9.

76. World Health Organization (WHO), Joint United Nations Programme on HIV/AIDS (UNAIDS). The Treatment 2.0 Framework for Action: Catalysing the Next Phase of Treatment, Care and Support. Geneva: WHO; 2011.

77. Coleman CI, Roberts MS, Sobieraj DM, Lee S, Alam T, Kaur R. Effect of dosing frequency on chronic cardiovascular disease medication adherence. Curr Med Res Opin. 2012;28(5):669-80.
78. Claxton AJ, Cramer J, Pierce C. A systematic review of the associations between dose regimens and medication compliance. Clin Ther. 2001;23(8):1296-310.

79. Reginster JY, Rabenda V, Neuprez A. Adherence, patient preference and dosing frequency: understanding the relationship. Bone. 2006;4(Suppl 1):S2-6.

80. Ma A, Chen DM, Chau FM, Saberi P. Improving adherence and clinical outcomes through an HIV pharmacist's interventions. AIDS Care. 2010;22(19):1189-94.

81. Kigozi IM, Dobkin LM, Martin JN, et al. Late-disease stage at presentation to an HIV clinic in the era of free antiretroviral therapy in sub-Saharan Africa. J Acquir Immune Defic Syndr. 2009;52(2):280-9.

82. Kitahata MM, Gange SJ, Abraham AG, et al. Effect of early versus deferred antiretroviral therapy for HIV on survival. N Engl J Med. 2009;360(18):1815-26.

83. Jani AV, Sitoe AE, Alfai ER, et al. Effect of point-of-care CD4 cell count tests on retention of patients and rates of antiretroviral therapy initiation in primary health clinics: an observational cohort study. Lancet. 2011;378(9802):1572-9.

84. Joint United Nations Programme on HIV/AIDS (UNAIDS). Breaking News Supplement: Meeting the Investment Challenge. Geneva: UNAIDS; 2012.

85. Torpey K, Kabaso M, Kasonde P, et al. Increasing the uptake of prevention of mother-to-child transmission of HIV services in a resource-limited setting. BMC Health Serv Res. 2010;10:29.

86. Killam WP, Tambatamba BC, Chintu N, et al. Antiretroviral therapy in antenatal care to increase treatment initiation in HIVinfected pregnant women: a stepped-wedge evaluation. AIDS. 2010;24(1):85-91.

87. Louwagie G, Girdler-Brown B, Odendaal R, Rossouw T, Johnson S, Van der Walt M. Missed opportunities for accessing HIV care among Tshwane tuberculosis patients under different models of care. Int J Tuberc Lung Dis. 2012;16(8):1052-8.

88. Tuller DM, Bangsberg DR, Senkungu J, Ware NC, Emenyonu N, Weiser SD. Transportation costs impede sustained adherence and access to HAART in a clinic population in southwestern Uganda: a qualitative study. AIDS Behav. 2010;14(4):778-84.

89. Hardon AP, Akurut D, Comoro C, et al. Hunger, waiting time and transport costs: time to confront challenges to ART adherence in Africa. AIDS Care. 2007;19(5):658-65.

90. Ware NC, Idoko J, Kaaya S, et al. Explaining adherence success in sub-Saharan Africa: an ethnographic study. PLoS Med. 2009;6(1):e1000011.

91. Wouters E, Van Damme W, van Rensburg D, Meulemans H. Impact of baseline health and community support on antiretroviral treatment outcomes in HIV patients in South Africa. AIDS. 2008;22(18):2545-8.

92. Decroo T, Telfer B, Biot M, et al. Distribution of antiretroviral treatment through self-forming groups of patients in Tete province, Mozambique. J Acquir Immune Defic Syndr. 2011;56(2): e39-44.

93. United Nations General Assembly. Political Declaration on HIV and AIDS: Intensifying Our Efforts to Eliminate HIV and AIDS. New York: United Nations General Assembly; 2011.

94. Joint United Nations Programme on HIV/AIDS (UNAIDS). Getting to Zero: 2011-2015 Strategy. Geneva: UNAIDS; 2010. 